CDD: 512.32

\title{
REMARKS ON ABSTRACT GALOIS THEORY ${ }^{1}$
}

\author{
NEWTON C. A. DA COSTA
}

Department of Philosophy

Federal University of Santa Catarina

$B R A Z I L$

ncacosta@usp.br

\section{OTÁVIO BUENO}

Department of Philosophy

University of Miami

Coral Gables, FL

USA

otaviobueno@mac.com

\begin{abstract}
This paper is a historical companion to a previous one, in which it was studied the so-called abstract Galois theory as formulated by the Portuguese mathematician José Sebastião e Silva (see da Costa, Rodrigues (2007)). Our purpose is to present some applications of abstract Galois theory to higher-order model theory, to discuss Silva's notion of expressibility and to outline a classical Galois theory that can be obtained inside the two versions of the abstract theory, those of Mark Krasner and of Silva. Some comments are made on the universal theory of (set-theoretic) structures.
\end{abstract}

Keywords: Galois theory. Structures. Models. Universal theory of structures. Mark Krasner. José Sebastião e Silva.

\section{INTRODUCTION}

In da Costa, Rodrigues (2007) the so-called abstract or generalized Galois theory, especially according to the views of José Sebastião e Silva, was presented. It was noted that there is another version of the

\footnotetext{
${ }^{1}$ We would like to thank Marcel Guillaume for extremely helpful comments on an earlier version of this paper. Thanks are also due to an anonymous referee for detailed suggestions that led to substantial improvements.
} 
theory - that of Mark Krasner - intrinsically linked to Silva's. In the present paper, we continue the study of these theories.

In particular, our main objectives are the following:

1. We consider some applications of generalized Galois theory to higher-order model theory and to the theory of species of structures (mathematical or axiomatic systems).

2. We analyze, from a set-theoretic stance, the notion of definability, in the wide sense of expressibility, a notion that is fundamental for Silva's theory.

3. We sketch the inter-connections between Silva's and Krasner's theories, showing in outline how classical Galois theory can be derived from both formulations of the abstract theory.

This paper is historical in nature, offering an outline of Silva's and Krasner's ideas and their significance. It is worth noting that analogs of Galois theory have been developed in many areas of mathematics (further details can be found, e.g., in: Krasner (1976), Erné et al. (1993), Mac Lane (1998), Davey, Priestley (2002), Gierz et al. (2002), Deneke et al. (2004), Picado (2005) and Galatos et al. (2007)). These approaches mesh with our generalized Galois theory, which is part of a universal theory of structures. However, this universal theory requires an extended set theory with universes, such as Zermelo-Fraenkel set theory with the axiom of choice (ZFC) together with universes plus a postulate to the effect that every set is contained in a universe. After all, several issues in a universal theory of structures cannot be accommodated in ZFC without universes, such as the Cartesian product of arbitrary families of structures. Thus, all of our constructions and results will be established in ZFC with universes (see Zakharov et al. (2006), Bunina, Zakharov (2006), da Costa (1972) and Brignole, da Costa (1971)). We shall also make free use of the terminology and the results of da Costa, Rodrigues (2007), without detailed comments. 


\section{HIGHER-ORDER STRUCTURES AND MODELS}

We define the set $T$ of types as follows:

1. The symbol $i$ belongs to $T$.

2. If $t_{0}, t_{1}, \ldots, t_{n-1} \in T$, then $\left\langle t_{0}, t_{1}, \ldots, t_{n-1}\right\rangle, 1 \leq n<\omega$, also belongs to $T$.

3. The elements of $T$ are only those given by clauses 1 and 2 .

The order of a type $t$, ord $(t)$, is introduced at follows:

1. $\operatorname{ord}(i)=0$.

2. $\operatorname{ord}\left(\left\langle t_{0}, t_{1}, \ldots, t_{n-1}\right\rangle\right)=\max \left\{\operatorname{ord}\left(t_{1}\right), \operatorname{ord}\left(t_{2}\right), \ldots, \operatorname{ord}\left(t_{n-1}\right)\right\}+1$.

If $D$ is a set, then it is possible to define a function $\tau_{D}$ or, to simplify, $\tau$, whose domain is $T$, by the following clauses:

1. $\tau(i)=D$.

2. If $t_{0}, t_{1}, \ldots, t_{n-1} \in T$, then $\tau\left(\left\langle t_{0}, t_{1}, \ldots, t_{n-1}\right\rangle\right)=\mathbb{P}\left(\tau\left(t_{1}\right) \times\right.$ $\left.\tau\left(t_{2}\right) \times \ldots \times \tau\left(t_{n-1}\right)\right)$, where $\mathbb{P}$ and $\times$ are the symbols for the power-set and the Cartesian product, respectively.

The set Urange $\left(\tau_{D}\right)$ is denoted by $\varepsilon(D)$, and is called the scale based on $D$. The objects of $\tau(i)$ are called individuals of $\varepsilon(D)$, and the objects of $\tau(t), \operatorname{ord}(t)>0$, are called objects or relations of type $t$; the type of individuals is $i$.

The cardinal $k(D)$, defined by the condition

$$
k(D)=\sup \{\overline{\bar{D}}, \overline{\overline{\mathbb{P}(D)}}, \overline{\overline{\mathbb{P}(\mathbb{P}(D))}}, \ldots\},
$$

is the cardinal associated to $\varepsilon(D)$. (Sometimes, instead of $k(D)$, we write $k_{D}$.)

A sequence is a function whose domain is an ordinal number, finite or infinite. $\hat{b}_{\lambda}$ is the range of the sequence $b_{\lambda}$. 
We call structure $e$ with basic set $D$ an ordered pair

$$
e=\left\langle D, r_{\iota}\right\rangle
$$

where $r_{\iota}$ is a sequence of elements of $\varepsilon(D)$. We also call $D$ the domain of $e$, and it is supposed to be non-empty. $D$ and the terms of $r_{\iota}$ are the primitive elements of $e$; the elements of $D$ are the individuals of $e$. Usually we identify such individuals with their unit classes. $\varepsilon(D)$ and $k(D)$ are the scale and the cardinal associated to $e$, respectively. In all cases, the ordinal which is the domain of $r_{\iota}$ is strictly less than $k(D)$.

The order of an object of $\varepsilon(D)$ is the order of its type. The order of $e=\left\langle D, r_{\iota}\right\rangle$, denoted by $\operatorname{ord}(e)$, is defined as follows: if there is the greatest order of the objects of the range of $r_{\iota}$, then $\operatorname{ord}(e)$ is this greatest order; otherwise, $\operatorname{ord}(e)=\omega$.

$L_{\omega \kappa}^{\omega}(R)$ is the higher-order infinitary language introduced in da Costa, Rodrigues (2007). Its blocks of quantifiers are finite, and conjunctions and disjunctions have length strictly less than $k$, when $k>\omega$; if $k=\omega$, such conjunctions and disjunctions are always finite. $R$ is the set of constants of the language, each having a fixed type.

$L_{\omega \kappa}^{\omega}(R)$ can be interpreted in structures of form

$$
e=\left\langle D, r_{\iota}\right\rangle
$$

the constants denoting $D$ and the objects $r_{\iota}$, each constant possessing the same type as that of the object it denotes. The sequence $r_{\iota}$ has as its domain an ordinal strictly less than $k_{D}$, usually finite or denumerable. In what follows we may identify a constant to the object it denotes; this will be done when there is no danger of confusion. In the structure $e=\left\langle D, r_{\iota}\right\rangle, D$ and the $r_{\iota}$ are called the primitive concepts or terms of $e$. 
Sentences of our language are formulas without free variables. If $\varphi$ is a sentence, then we say that $\varphi$ is true in the structure

$$
e=\left\langle D, r_{\iota}\right\rangle
$$

in analogy with the case of first-order (finitary) logic. To express that $\varphi$ is true in $e$ we write:

$$
e \vDash \varphi .
$$

The notions of model of a set of sentences and model of a sentence are the usual ones. We write $\Gamma \vDash \varphi$ to mean that every model of the set of sentences $\Gamma$ is also a model of $\varphi$.

Let $a$ be an object of $\varepsilon(D)$, that is, of the scale associated to $e$. In da Costa, Rodrigues (2007), using the language $L_{\omega \kappa}^{\omega}(R)$, it was studied the concept of definability (in the wide sense) of an element of $\varepsilon(D)$ in the structure $e$ by means of the sequence $b_{\lambda}$ of elements of $\varepsilon(D)$. This concept is characterized as follows:

$a$ is definable in the wide sense in $e$ with the help of the sequence $b_{\lambda}$ if there is a formula $\varphi\left(x ; b_{\lambda}\right)$ of $L_{\omega \kappa}^{\omega}\left(R \cup \hat{b}_{\lambda}\right)$ such that

$$
e \vDash \forall x\left(x=a \leftrightarrow \varphi\left(x ; b_{\lambda}\right)\right),
$$

where $\varphi\left(x ; b_{\lambda}\right)$ contains $x$ as its only free variable.

Evidently, it is supposed that $L_{\omega \kappa}^{\omega}(R)$ is interpreted in $e$, and that other standard conditions are satisfied.

Now we introduce the concept of definability in the wide sense on the basis of the set $\Gamma$ of sentences and in terms of a finite sequence of constants (or a set of constants). For that, let $\Gamma\left(r, b_{\lambda}, c_{0}, c_{1}, \ldots, c_{n-1}\right)$ be a set of sentences in which the only constants are $r$, the terms of the sequence $b_{\lambda}$ and the terms of the finite sequence $c_{0}, c_{1}, \ldots, c_{n-1}$. (We will abbreviate that set by $\Gamma$.) The constant $r$ is said to be definable in the wide sense on the basis of the set $\Gamma$ by means of the constants 
$b_{\lambda}$ if there is a formula $\psi\left(x ; b_{\lambda}\right)$, having $x$ as its sole free variable and containing as constants only the constants $b_{\lambda}$, such that

$$
\Gamma \vDash \forall x\left(x=r \leftrightarrow \psi\left(x ; b_{\lambda}\right)\right) .
$$

Clearly, $x$ and $r$ must have the same type. (From now on, restrictions of type will not be made explicit whenever there is no danger of confusion.)

When in $L_{\omega \kappa}^{\omega}(R), \kappa=\omega$, we say that $r$ is strictly definable on the basis of $\Gamma$ by means of the constants $b_{\lambda}$-a notion that we essentially owe to Tarski (see Krasner (1968-69), chapter X). Of course, throughout our discussion, we suppose that our language is interpreted in suitable structures. Normally, we take $\kappa=k(D)$.

Theorem 1.1 Under the above conditions, $r$ is definable in the wide sense on the basis of $\Gamma$ by means of the constants $b_{\lambda}$ if and only if, in every model of $\Gamma$, the object denoted by $r$ is definable in the wide sense in terms of the sequence $b_{\lambda}$ of elements of the model.

Proof Implication from left to right: If $r$ is definable in the wide sense on the basis of $\Gamma$ by means of $b_{\lambda}$, then evidently the object denoted by $r$, in any model of $\Gamma$, is definable in the wide sense in terms of the objects $b_{\lambda}$ in such a model.

Implication from right to left: If $r$ is definable in the wide sense in every model $e$ of $\Gamma$ in terms of $b_{\lambda}$, then there is in $e$ one and only one object that is denoted by $r$. This means that we have

$$
e \vDash \exists ! x \tilde{\Gamma}\left(x ; b_{\lambda}, c_{0}, c_{1}, \ldots, c_{n-1}\right),
$$

where $\tilde{\Gamma}$ is the conjunction of the formulas of $\Gamma$ in which $r$ is replaced by the new variable $x$. Therefore,

$$
e \vDash \forall x\left(x=r \leftrightarrow \tilde{\Gamma}\left(x ; b_{\lambda}, c_{0}, c_{1}, \ldots, c_{n-1}\right)\right)
$$


and, as a result,

$$
e \vDash \exists x_{0} \exists x_{1} \ldots \exists x_{n-1} \forall x\left(x=r \leftrightarrow \tilde{\Gamma}\left(x ; b_{\lambda}, x_{0}, x_{1}, \ldots, x_{n-1}\right)\right) .
$$

So,

$$
e \vDash \forall x \exists x_{0} \exists x_{1} \ldots \exists x_{n-1}\left(x=r \leftrightarrow \tilde{\Gamma}\left(x ; b_{\lambda}, x_{0}, x_{1}, \ldots, x_{n-1}\right)\right)
$$

and

$$
e \vDash \forall x\left(x=r \leftrightarrow \exists x_{0} \exists x_{1} \ldots \exists x_{n-1} \tilde{\Gamma}\left(x ; b_{\lambda}, x_{0}, x_{1}, \ldots, x_{n-1}\right)\right) .
$$

Hence, the formula

$$
\exists x_{0} \exists x_{1} \ldots \exists x_{n-1} \tilde{\Gamma}\left(x ; b_{\lambda}, x_{0}, x_{1}, \ldots, x_{n-1}\right)
$$

defines $r$ in the wide sense on the basis of $\Gamma$ by means of the constants $b_{\lambda}$.

Corollary Under the hypotheses of the theorem, $r$ is definable on the basis of $\Gamma$ by means of $b_{\lambda}$ iff in any model e of $\Gamma$ the object $r$ is invariant under the Galois group of the restriction $e^{\prime}$ of $e$ to the language without the constant denoting $r$.

Proof Consequence of the theorem 6.5 of da Costa, Rodrigues (2007).

Theorem 1.2 Under the preceding conditions, let $u$ and $v$ be two new constants added to our language. Then, in order for $r$ to be definable in the wide sense on the basis of $\Gamma$ by means of $b_{\lambda}$, it is necessary and sufficient that

$$
\begin{aligned}
& \exists x_{0} \exists x_{1} \ldots \exists x_{n-1} \tilde{\Gamma}\left(u ; b_{\lambda}, x_{0}, x_{1}, \ldots, x_{n-1}\right) \wedge \\
& \exists x_{0} \exists x_{1} \ldots \exists x_{n-1} \tilde{\Gamma}\left(v ; b_{\lambda}, x_{0}, x_{1}, \ldots, x_{n-1}\right) \vDash u=v .
\end{aligned}
$$

Manuscrito - Rev. Int. Fil., Campinas, v. 34, n. 1, p. 151-183, jan.-jun. 2011. 
Proof The condition is sufficient: the expression above implies that any model $e$ of $\Gamma$ is such that

$$
e \vDash \exists ! x \exists x_{0} \exists x_{1} \ldots \exists x_{n-1} \tilde{\Gamma}\left(x ; b_{\lambda}, x_{0}, x_{1}, \ldots, x_{n-1}\right),
$$

which entails that $r$ denotes in $e$ an object that is definable in the wide sense in this model in terms of $b_{\lambda}$. Hence, by Theorem 1 , the formula

$$
\exists x_{0} \exists x_{1} \ldots \exists x_{n-1} \tilde{\Gamma}\left(x ; b_{\lambda}, x_{0}, x_{1}, \ldots, x_{n-1}\right)
$$

clearly defines $r$ in the wide sense on the basis of $\Gamma$ by means of $b_{\lambda}$.

The condition is necessary: the proof is immediate.

Corollary Under the hypotheses of the theorem, $r$ is definable on the basis of $\Gamma$ by means of $b_{\lambda}$ iff in any model e of $\Gamma$, the formula

$$
\exists x_{0} \exists x_{1} \ldots \exists x_{n-1} \tilde{\Gamma}\left(x ; b_{\lambda}, x_{0}, x_{1}, \ldots, x_{n-1}\right)
$$

defines in the wide sense, in $e$, in terms of $b_{\lambda}$, an element that remains invariant under the group of automorphisms of e that preserve the $b_{\lambda}$.

Proof Similar to the proof of the corollary to the previous theorem.

The preceding discussion extends some theorems obtained by Tarski in Tarski (1983), chapter X; the other theorems of that chapter can also be extended in the same vein, including Tarski's higher-order version of Padoa's method.

Given structures $e_{1}=\left\langle D_{1}, r_{\iota}\right\rangle$ and $e_{2}=\left\langle D_{2}, s_{\iota}\right\rangle$, we call bijection between $e_{1}$ and $e_{2}$ any bijection of $D_{1}$ on $D_{2}$, canonically extended to a bijection of $\varepsilon\left(D_{1}\right)$ on $\varepsilon\left(D_{2}\right)$.

Let $\varphi$ be a bijection between two models $e_{1}$ and $e_{2}$ of the set $\Gamma$ of sentences. We say that $\varphi$ is a $u$-isomorphism between $e_{1}$ and $e_{2}$ if $\varphi$ is 
an isomorphism between the restrictions of $e_{1}$ and $e_{2}$ to the language having $u$ as its only constant.

We consider now a set of sentences $\Gamma\left(v, b_{\mu}, c_{\nu}\right)$ composed by formulas having as their only constants $v$ and the elements of range $\left(b_{\mu}\right) \cup$ range $\left(c_{\nu}\right)$, all of them supposed to be distinct and $c_{\nu}$ finite. The conjunction of the formulas of $\Gamma\left(v, b_{\mu}, c_{\nu}\right)$ will be designated by $\tilde{\Gamma}\left(v, b_{\mu}, c_{\nu}\right)$. Replacing in $\tilde{\Gamma}\left(v, b_{\mu}, c_{\nu}\right)$ the constants $c_{\nu}$ by distinct new variables $x_{\nu}, \exists x_{\nu} \tilde{\Gamma}\left(v, b_{\mu}, x_{\nu}\right)$ will be the existential quantification of $\tilde{\Gamma}\left(v, b_{\mu}, x_{\nu}\right)$ by the block of existential quantifiers $\exists x_{\nu}$. Instead of $\Gamma\left(v, b_{\mu}, c_{\nu}\right)$ we will write simply $\Gamma$.

The proposition below is a generalization of Shoenfield's version of the theorem of Beth (Shoenfield (1967), p. 81-82).

Theorem 1.3 Let $\Gamma$ be a set of formulas as described above. We have: $v$ is definable in the wide sense on the basis of $\Gamma$ by means of the constants $b_{\mu}$ iff for every pair of models of $\Gamma e_{1}=\left\langle D_{1}, r_{\iota}\right\rangle$ and $e_{2}=\left\langle D_{2}, s_{\iota}\right\rangle$, and every bijection $\varphi$ between $e_{1}$ and $e_{2}$ which is a u-isomorphism for all $u$ in range $\left(b_{\mu}\right), \varphi$ is also a v-isomorphism.

Proof If $v$ is definable in the wide sense on the basis of $\Gamma$ by means of $b_{\mu}$, then clearly any two models $e_{1}$ and $e_{2}$ of $\Gamma$ satisfy the condition on $u$-isomorphisms and $v$-isomorphisms of the theorem.

On the other hand, if the condition is verified, then for any model $e$ of $\exists x_{\nu} \tilde{\Gamma}\left(v, b_{\mu}, x_{\nu}\right)$, the automorphisms of $e$ that preserve $u$, for any $u$ in range $\left(b_{\mu}\right)$, also preserve $v$. So, $v$ is definable in the wide sense in $e$ on the basis of $\exists x_{\nu} \tilde{\Gamma}\left(v, b_{\mu}, x_{\nu}\right)$ by means of $b_{\mu}$, taking into account Theorem 6.5 of da Costa, Rodrigues (2007) and Theorem 1.1 above. Therefore, $v$ is also definable in the wide sense on the basis of $\Gamma$ by means of $b_{\mu}$.

Let $e$ be a structure, $D$ its domain, and $G$ its Galois group. Two elements $x$ and $y$ of $D$ are said to be $G$-equivalent if there is $g$ in $G$ such that $y=g x$. This relation determines a partition in $D$, and its 
equivalence classes are the orbits in $e$. (Orbits are the irreducible sets of da Costa, Rodrigues (2007).)

The languages $L_{\omega \omega}^{n}(R), L_{\omega \kappa}^{n}(R)$ and $L_{\kappa \kappa}^{n}(R), 1 \leq n<\omega$, are called languages of order $n$; they contain variables and constants of order $<n$ and constants of order $n$, and their semantics are easily constructed. $L_{\omega \omega}^{\omega}(R), L_{\omega \kappa}^{\omega}(R)$ and $L_{\kappa \kappa}^{\omega}(R)$ are called languages of order $\omega$. Obvious logics correspond to all these languages.

If $e=\left\langle D, r_{\iota}\right\rangle$, with $D$ infinite, is a structure of order $n, 1 \leq n<\omega$, in which $L_{\omega \omega}^{n}(R), L_{\omega \kappa}^{n}(R)$ or $L_{\kappa \kappa}^{n}(R)$ is interpreted, then the cardinal $k(e, n)$, associated to the structure $e$, is defined as follows:

$$
k(e, n)=\operatorname{card}\left(\mathbb{P}^{n}(D)\right),
$$

where the power-set operator $\mathbb{P}$ is applied $n$ times. When $D$ is finite, $k(e, n)=\aleph_{0}$. Usually, we denote $k(e, n)$ by $\kappa$.

Examining the proofs of Caulton, Butterfield (2008), one verifies that most results, with convenient adaptations, remain valid for logics of order $n, 1 \leq n<\omega$, and their corresponding structures. For example, we have:

1. If $e=\left\langle D, r_{\iota}\right\rangle$ is a structure of order $n, 1 \leq n<\omega$, then the element $r$ of $\varepsilon(D)$ whose order is $m, 1 \leq m \leq n$, is invariant under the Galois group of $e$ iff $r$ is definable in $L_{\omega \kappa}^{l}\left(\hat{r}_{\iota}\right)$, for $\kappa=k(e, n)$ and some $l$ such that $n \leq l<\omega$.

2. Given the $n$-order structure $e=\left\langle D, r_{\iota}\right\rangle$, the element $r \in \varepsilon(D)$, of order $\leq n$, is definable in the wide sense in $L_{\kappa \kappa}^{n}\left(\hat{r}_{\iota}\right), \kappa=k(e, n)$, iff $r$ is invariant under the automorphisms of the Galois group of $e(1 \leq n<\omega)$. (Generalization of a theorem of Rogers; see Galatos et al. (2007).)

3. Theorem 1.3 is true for structures of order $n$ in $L_{\omega \kappa}^{n}(R)$, in $L_{\omega \omega}^{n}(R)$, and, for $c_{\nu}$ infinite, in $L_{\kappa \kappa}^{n}(R), 1 \leq n<\omega$. 
Two structures $e_{1}$ and $e_{2}$ for the same language are called equipolent if every sentence of the language which is true in $e_{1}$ is also true in $e_{2}$ and conversely.

Theorem 1.4 Let $e=\left\langle D, r_{\iota}\right\rangle$ and $e^{\prime}=\left\langle D^{\prime}, r_{\iota}^{\prime}\right\rangle$ be two similar structures in which $L_{\omega \theta}^{\omega}(R)$ is interpreted, where $\theta$ is the greatest of the cardinals associated with $e$ and $e^{\prime}$. Then $e$ and $e^{\prime}$ are equipolent (in relation to $\left.L_{\omega \theta}^{\omega}(R)\right)$ iff there exists a bijection between the set of orbits of $e$ and the set of orbits of $e^{\prime}$, such that the corresponding orbits are determined by the same irreducible formula.

Proof An open formula of the language $L_{\omega \theta}^{\omega}(R)$, interpreted in a suitable structure, is called irreducible if it defines in the wide sense an orbit of the structure (see Caulton, Butterfield (2008)).

If $e$ and $e^{\prime}$ are structures in the conditions of the theorem, then it is easy to show that, in $e$ and $e^{\prime}$, every open formula is equivalent to the disjunction of the same family of irreducible formulas. So, reasoning by induction on the number of logical operators appearing in a given open formula, it follows that every open formula that is satisfiable in $e$ is also satisfiable in $e^{\prime}$, and conversely. Hence, any sentence that is true in $e$ (or in $e^{\prime}$ ) is also true in $e^{\prime}$ (or in $e$, respectively).

Theorem 1.5 Under the conditions of Theorem 1.4, $e$ and $e^{\prime}$ are isomorphic iff their corresponding orbits have the same cardinal.

Proof It suffices to note that any atomic formula is, in both structures, the disjunction of the same family of irreducible formulas. Therefore, if $\varphi$ is the bijection between the domains of $e$ and $e^{\prime}$, expanding the bijections to their orbits, $\left\langle a_{1}, a_{2}, \ldots, a_{l}\right\rangle$ satisfies a first-order relation of $e$ of degree $l$ iff $\left\langle\varphi\left(a_{1}\right), \varphi\left(a_{2}\right), \ldots, \varphi\left(a_{l}\right)\right\rangle$ also satisfies the corresponding relation of $e^{\prime}$. We treat similarly the case of higher-order relations. 
Theorems 1.4 and 1.5 are true for structures of order $n$ and language $L_{\omega \theta}^{n}(R), 1 \leq n \leq \omega$.

We also have:

Theorem 1.6 Given the structure e of order $n, 1 \leq n \leq \omega$, there exists a finite number of first-order relations that, added to e, transform e in a rigid structure.

Part of the previous discussion, such as the results connected with Theorem 1.1 and its corollary, can be adapted to the case of species of structures (in Bourbaki's sense; see Bourbaki (1968)), theories or axiomatic systems. From this perspective, for example, Theorem 1.1 relates species of structures to their models.

Consider the following two propositions:

(I) If $X$ is irreducible, then $X$ is an orbit.

and

(II) The element $r$ of $\varepsilon(D)$ is invariant under the Galois group of $e=\left\langle D, r_{\iota}\right\rangle$ iff $r$ is definable in the wide sense (or expressible). (Silva's theorem.)

In da Costa, Rodrigues (2007), proposition (I) was proved with the help of (II). However, we observe that (I) can be established without the use of (II), and that Silva's theorem is a direct consequence of (I).

We also have:

Theorem 1.7 The element $r$ of $\varepsilon(D)$ is definable in the wide sense iff $\{r\}$ is an orbit.

Proof Immediate.

Theorem 1.8 Consider the structure $e=\left\langle D, r_{\iota}\right\rangle$. Let I be the Galois group of $e$, and let $\Delta_{\alpha}, \alpha \in A$, be the family of the restrictions of I to 
the orbits of D. It follows that, in $e$,

$$
\overline{\bar{I}}=\prod_{\alpha \in A} \overline{\bar{\Delta}} \alpha .
$$

Proof Immediate.

Note that the previous theorem remains valid for whatever type.

To finish this section, we present an interesting, well-know fact concerning Galois groups (of structures). Let $e=\left\langle D, r_{\iota}\right\rangle$ be a firstorder structure in which $L_{\omega \omega}^{1}\left(\tilde{r}_{\iota}\right)$ is interpreted. An $n$-type, according to usual model theory (see Shoenfield (1967), chapter V), determines an orbit on $D^{n}, 1 \leq n<\omega$. We say that $e$, in the language $L_{\omega \omega}^{1}\left(\tilde{r}_{\iota}\right)$, is countably categorical or $\omega$-categorical, if any first-order structure with countably infinite domain and which satisfies the same first-order sentences as $e$ is isomorphic to $e$. Then the Ryll-Nardzewski theorem (see Shoenfield (1967, chapter V)) can be reformulated as follows:

Theorem 1.9 The first-order structure $e=\left\langle D, r_{\iota}\right\rangle$, with $D$ countably infinite, is $\omega$-categorical iff its Galois group determines on $D^{n}$ a finite number of orbits, $1 \leq n<\omega$.

\section{AN OUTLINE OF KRASNER'S THEORY}

In this section, we outline Krasner's theory, showing how it can be subsumed under the framework of Silva's theory. Further details on Krasner's theory can be found in Krasner (1938), Krasner (1950), Krasner (1968-69), and Krasner (1973). Our summary of the theory is based, in particular, on Krasner (1968-69), and Krasner (1973).

Krasner summarizes the principal problems of classical Galois theory as follows:

Let $k$ be a field and $K$ an algebraic extension of $k$. We shall suppose that $K / k$ is of finite degree, although this 
hypothesis is not completely necessary for all results. We shall also suppose that $K / k$ is normal; i.e. every irreducible polynomial with coefficients in $k$, which has one root in $K$, can be completely decomposed in $K$ in linear factors, so 'has all its roots in $K$ ' [...]

The automorphisms of $K / k$ form a group with respect to the usual composition of mappings; this group is called the Galois group of the extension $K / k$ and is denoted by $g_{K / k}$. If $A$ is a subset of $K$, we consider the part of $g_{K / k}$ which preserves every element of $A$ (i.e. consists of the $\sigma \in g_{K / k}$ which satisfy $\sigma \bar{a}=a$ for all $a \in A$ ). This part of $g_{K / k}$ is a subgroup of $g_{K / k}$, denoted by $g_{K / k}^{(A)}$. Any subgroup of $g_{K / k}$, which is $g_{K / k}^{(A)}$ for some $A \subseteq K$, is called a Galoisian subgroup of $g_{K / k}$.

The main problems of classical Galois theory can be formulated as follows:

1. Given $A \subseteq K$, what is the set $\bar{A}$ of all elements $b \in K$ which are preserved by the elements of $g_{K / k}^{(A)}$ (i.e. are such that $\sigma b=b$ for all $\left.\sigma \in g_{K / k}^{(A)}\right)$ ?

2. What subgroups of $g_{K / k}$ are Galoisian?

Classical Galois theory gives the following answers to these problems:

1. $\bar{A}$ is the closure of $A \cup k$ with respect to the operations $x+y, x y, x^{-1}$ and, if the characteristic of $k$ is $p \neq 0$, the operation $\sqrt[p]{x}$ in case $\sqrt[p]{x} \in K$ (i.e. if $x \in \bar{A}$ and $\sqrt[p]{x} \in K$, then $\sqrt[p]{x} \in \bar{A})$. Note that $-1 \in k$, so $x+y$ includes the case of $x-y$.

2. Every subgroup of $g_{K / k}$ is Galoisian. 
From the answer to the first problem we see that, when $A$ ranges over all subsets of $K, \bar{A}$ ranges over the intermediate fields between $k$ and $K$ which are perfect in $K$ (i.e. fields $L$ with $k \subseteq L \subseteq K$ and such that if the $p$-th root of an element of $L$ is in $K$, it is also in $L$ ).

The answer (1) is valid in two more general cases: the case when the extension $K / k$ is algebraic and normal (without the hypothesis "of finite degree") and the case when the extension is algebraically closed without being algebraic. For the answer (2), the hypothesis "of finite degree" is needed. (Krasner (1973), p. 15-17.)

Krasner introduces a new kind of first-order infinitary structure and studies the corresponding two central problems which are generalizations of those of classical Galois theory. His theory is outlined in what follows, and some of its applications are discussed.

Let us consider two sets $E$ and $X$, the first is called the support set and the second is called the auxiliary set of arguments or of variables. An $X$-point of $E$ is any mapping $P: X \rightarrow E$. An $X$-relation on $E$ is any set $r \subset E^{X}$. So, an $X$-relation on $E$ is a set of $X$-points of $E$. We suppose that $X$ and $E$ are not empty and that $X \cap E=\emptyset$; in addition, the cardinal of $X$ is strictly greater than, or equal to, the cardinal of E.

Given $X^{\prime}$ such that $X \cap X^{\prime}=\emptyset$, we identify the $X$-relation (on $E$ ) $r$ to the $X \cup X^{\prime}$-relation (on $E$ ) $r \times E^{X^{\prime}}$, prolonging the identification by symmetry and transitivity.

The following operations are central in Krasner's theory ( $X$-relation on $E$ will be abbreviated simply as $X$-relation):

Intersection of any sequence $R$ of $X$-relations, operation that generates another $X$-relation, and is denoted by $\cap R$. 
The complement of an $X$-relation $r: \tilde{r}=E^{X}-r . \tilde{r}$ is also denoted by $\sim r$.

(Clearly, the union of a sequence $R$ of $X$-relations is definable in terms of $\cap$ and $\sim$.)

Cylindrification: If $X^{\prime} \subset X$, then a cylindrification associates to any $X$-relation $r$ another $X$-relation $r_{X^{\prime}}=$ $\left(r / X^{\prime}\right) \times E^{X-X^{\prime}}$, where $r / X^{\prime}$ means the restriction of $r$ to $X^{\prime}$. In other words, an $X$-point $P$ belongs to $r_{X^{\prime}}$ iff there exists an $X$-point $P^{\prime}$ in $r$ such that $P$ coincides with $P^{\prime}$ on $X^{\prime}$.

$r$ is said to be identical on $X-X^{\prime}$, or $r$ is said to be not dependent of $X-X^{\prime}$, iff $r=r_{X^{\prime}}$.

$r_{X^{\prime}}$ is also denoted by $\exists X^{\prime} r$.

The fourth fundamental operation is mutation or change of variables, which is of two kinds:

Simple mutations: Let $X^{\prime}$ and $X^{\prime \prime}$ be two subsets of $X$ having the same cardinal, and let $f$ be a bijection of $X^{\prime}$ on $X^{\prime \prime}$. If $r$ is a relation identical on $X-X^{\prime}$, then the mutation of $r$ by $f$ is the $X$-relation $r^{(f)}$ satisfying the condition that an $X$-point $P$ belongs to $r^{(f)}$ iff there exists $P^{\prime}$ in $r$ such that $P(f(x))=P^{\prime}(x)$ for every $\mathrm{x}$ in $X^{\prime}, r^{(f)}$ being identical outside of $X^{\prime \prime}$ (or on $X-X^{\prime \prime}$ ).

Generalized mutations: We suppose that $X^{\prime}$ and $X^{\prime \prime}$ are two subsets of $X$ and that $C^{\prime}$ and $C^{\prime \prime}$ are equivalences on $X^{\prime}$ and $X^{\prime \prime}$ respectively, and that the classes of equivalence $X^{\prime} / C^{\prime}$ and $X^{\prime \prime} / C^{\prime \prime}$ possess the same cardinal. We denote by $f$ a bijection between $X^{\prime} / C^{\prime}$ and $X^{\prime \prime} / C^{\prime \prime}$. On the other hand, $Y$ being a subset of $X$ and $C$ an equivalence on $Y$, an $X$-relation $s$ is said to be compatible with $C$ if $s$ is identical on $X-Y$ and if, for any $x \in Y$ and any $P \in s$, 
$\mathrm{P}(x)$ only depends of the class of equivalence of $Y / C$ to which $x$ belongs. Then, returning to the $X$-relation $r$, if $r$ is compatible with $C^{\prime}$ on $X^{\prime}$ and identical out of $X^{\prime}$, the generalized mutation of $r$ by $f$, noted $r^{(f)}$, is the $X$ relation constituted by the points $P$ such that there exists $P^{\prime} \in r$ verifying the condition that for all $M \in X^{\prime} / C^{\prime}$, $\mathrm{P}[f(M)]=\mathrm{P}^{\prime}[M]$, where $\mathrm{P}[f(M)]$ is the value assumed by $P$ in any element of $f(M)$ and $\mathrm{P}^{\prime}[M]$ is the value that $P^{\prime}$ assumes in any element of $M$.

Mutations are partial operations, but it is not difficult to transform them into total operations.

There are infinitely many operations of intersection (and union), each of them characterized by the ordinal of the family of $X$-relations to which they are applied, that is, by its arity. Normally the operations of intersection (and union) are here disposed in a sequence whose domains are ordinals strictly less than a fixed cardinal; in the case of Krasner structures, structures to be introduced below, the cardinal is the cardinal associated with the structure. The ordinals of the sequence are the arities of the operations of intersection (or union). This sequence of intersections will be denoted by $\sqcap$ (and of unions by $\sqcup$ ), which is clearly an abuse of language. Given $X$ and $E$, cylindrifications and mutations can also be disposed in sequences, sequences which will be denoted by $\exists$ and $V$, respectively. So, the concatenation of the sequences $\sim$ (an unary sequence), $\sqcap, \exists$ and $V$ may be written

$$
\sim, \sqcap, \exists, V
$$

where the comma means concatenation.

Let $r_{\lambda}$ be a sequence of $X$-relations on $E, D=X \cup E$, with $X \sqcap E=\emptyset$ and $\operatorname{card}(X) \geq \operatorname{card}(E), x_{\mu}$ a sequence of all elements of $X$ without repetitions, and 


$$
x_{\mu}, r_{\lambda}, \sim, \sqcap, \exists, V
$$

the concatenation of such sequences. We now define a Krasner structure (or $K$-structure) as a set-theoretic construct of the following form:

$$
K=\left\langle D, x_{\mu}, r_{\lambda}, \sim, \sqcap, \exists, V\right\rangle .
$$

Clearly, $K$ is a structure in the sense of section 1 of da Costa, Rodrigues (2007); hence, the generalized Galois theory of that paper can be applied to $K$. In particular, since $x_{\mu}$ is a sequence of primitive relations (0-adic relations) of $K$, any automorphism of $K$ must leave invariant the members of $X$. It is also clear that the automorphisms of

$$
\tilde{K}=\left\langle D, x_{\mu}, r_{\lambda}\right\rangle
$$

are the same as those of $K$. Moreover, taking into account that the fundamental operations are invariant under the Galois group of $K$, they are logically definable (see da Costa, Rodrigues (2007)). Therefore, $K$ and $\tilde{K}$ are equivalent structures.

As in da Costa, Rodrigues (2007), all sequences concerning a fixed $K$-structure have domains which are ordinals strictly less than the cardinal associated with the structure.

An $X$-relation $r$ is said to be $K$-definable in the Krasner structure $K$ if and only if:

1. $r$ is one of the $X$-relations $r_{\lambda}$; or

2. $r$ can be obtained from a $K$-definable $X$-relation by one of the operations $\sim, \exists$ or $V$; or

3. $r$ is the intersection of a sequence of $K$-definable $X$-relations; and

4. $r$ is $K$-definable only in the cases $1-3$. 


\section{Examples}

Any $r_{\lambda}$ is $K$-definable in the structure $K ; \emptyset$ and $I=E^{X}$ are always $K$-definable.

A set $S$ of $X$-relations is called $K$-closed iff any $X$-relation $K$ definable in a structure like $\tilde{K}$ above, where $r_{\lambda}$ is a sequence of all elements of $S$, belongs to $S$. The Krasner closure or $K$-closure of $S$, denoted by $S^{(K)}$, is the intersection of all $K$-closed sets containing $S$.

Theorem 2.1 Let $S \cup\{r\}$ be a set of $X$-relations. Then $r \in S^{(K)}$ implies that $r$ is definable in the wide sense (or expressible) in the structure

$$
K^{\prime}=\left\langle D, x_{\mu}, r_{\lambda}\right\rangle
$$

where $r_{\lambda}$ is a sequence of all members of $S$.

Proof The operations $\sim, \exists, V$ and $\sqcap$ are invariant under the Galois group of $K^{\prime}$, as is easy to verify. Therefore, by Theorem 6.5 of da Costa, Rodrigues (2007), they are definable in the wide sense in $K^{\prime}$. This fact entails that $r$ is expressible in $K^{\prime}$.

Two $K$-structures $K_{1}=\left\langle E \cup X, x_{\mu}, r_{\lambda}\right\rangle$ and $K_{2}=\left\langle E \cup X, x_{\mu}, r_{\eta}\right\rangle$ are said to be $K$-equivalent if the $K$-closures of the $X$-relations of $K_{1}$ and of the $X$-relations of $K_{2}$ are equal.

Theorem 2.2 [Krasner] Two $K$-structures are $K$-equivalent iff they have the same Galois group.

Proof The result is established by employing the notion of orbit of the $X$-points of both structures, as well as the properties of the fundamental operations (for details, see Krasner (1950) and Krasner (1973)).

Corollary 1. An X-relation $r$ is $K$-definable in a $K$-structure iff $r$ is invariant under the action of the automorphisms of the Galois group of the $K$-structure. 
Corollary 2. The converse of Theorem 2.1 is true.

Theorem 2.3 [Krasner] Given any group of permutations $G$ of set $E$, there exists a K-structure with support $E$ and whose Galois group is $G$.

Proof See Krasner (1950) and Krasner (1973).

We introduce as usual the concept of an endomorphism of a $K$ structure. The semi-group of the endomorphisms of a $K$-structure is called the Galois semi-group of the structure. Krasner elaborated a theory of the endomorphisms of $K$-structures similar to his extension of Galois theory (that concerns automorphisms). Propositions similar to the theorems of this section can be established (see Krasner (196869) and Krasner (1973)). As noted in da Costa, Rodrigues (2007), Silva also sketches the development of a theory of endomorphisms, correlated with his formulation of the abstract Galois theory. In a wide sense, the generalized Galois theory includes both automorphisms and endomorphisms.

Krasner studied some special cases of $K$-structures, named by him eliminative structures. By means of theses structures it is possible to establish the basic theorems of classical Galois theory, as well as the central theorems of other theories, for instance, Picard's version of Galois theory for differential equations.

It should be recalled that one of the main results of classical Galois theory is the proposition asserting that there are certain connections between subfields of algebraic extensions of a field and the subgroups of the associated Galois group. In other words, classical Galois theory is a theory of the duality between groups of permutations and certain of their invariants; these invariants are finitary relations defined by means of the operations of fields. Krasner generalized this situation by considering infinitary relations and the corresponding abstract structures. 
When the infinitary relations depend only on a finite number of coordinates, we obtain the ordinary case of finitary relations and operations. Thus, classical Galois theory can be subsumed under the abstract or generalized Galois theory.

The similarity between abstract Galois theory and the Erlangen Program is obvious; in fact, the latter can be viewed as a part of the former. (We will return to this point below.) Moreover, generalized Galois theory presents strong connections with infinitary languages and cylindric algebras. (For various applications of Krasner's theory, see, for instance, Krasner (1968-69, 1973 and 1976).)

\section{SILVA'S OPERATIONAL GALOIS THEORY}

Silva showed that his general theory (Sebastião e Silva (1985, 1945 and 1985)) can be adapted to obtain classical Galois theory. Let us see how this is done.

In this section, we will be concerned with structures of the following form:

$$
e=\left\langle D, o_{\iota}\right\rangle
$$

where $o_{\iota}, \iota \in \lambda$, are first-order operations (or operators), and $\lambda$ is a finite ordinal. The operations $o_{\iota}$ associate elements of $D$ with $n$-tuples of elements of $D ; n, 0 \leq n<\omega$, is the rank of the operator.

$L=L\left(o_{\iota}\right)$ is a first-order language whose only nonlogical primitive symbols are $o_{\iota}$. Terms and formulas are defined as usual, starting with variables and the operators $o_{\iota} . L$ is supposed to be interpreted in a structure of the above form.

If $V$ and $U$ are subsets of $D$, we say that $v \in V$ is operationally definable in terms of $U$ if $v=a$ is true, where the term $a$ is composed by the operators $o_{\iota}$ and elements of $U$. Similarly, we define the concept of an operator operationally definable in terms of a set of operators and a set of elements of $D$. 
An operational relation is a relation characterized by a formula of the form

$$
a=b,
$$

where $a$ and $b$ are terms of $L$. If such a formula has $n$ free variables, then the relation is of rank or of degree $n, 0 \leq n<\omega$.

Similarly to da Costa, Rodrigues (2007), we introduce the notions of operationally closed set and operational base of a set $V$ with reference to another set $W$, such that $W \subset V \subset D$. We will refer to the base of $V$ with reference to $W$ by 'base of $V / W$ '.

For instance, in $e=\left\langle D, o_{\iota}\right\rangle, D$ is always operationally closed, and if all $o_{\iota}$ have rank $>0$, the empty set is also operationally closed. The operationally closed sets of a group are its subgroups, and the operationally closed subsets of a field are its subfields.

In the case of fields, it is possible to define, operationally, sum and product in terms of difference and quotient, but not conversely. A subfield of a field may be operationally closed though not algebraically closed. The operators or functions operationally definable in the elements of a field $K$ are the rational functions whose coeficients are in $K$, and the relations operationally definable in terms of $\mathrm{K}$ are the algebraic equations with coeficients in $K$.

The definition of irreducible operational relation is formulated as in da Costa, Rodrigues (2007). However, if $\rho$ is an operational relation which is satisfied by an $n$-tuple $d$, we cannot guarantee that there exists an irreducible operational relation $\rho^{\prime}$ such that $d \in \rho^{\prime}$ and $\rho^{\prime} \subset \rho$.

The concepts of operationally strong and operationally weak isomorphisms can be introduced in analogy with da Costa, Rodrigues (2007). However, these concepts coincide, and we are allowed simply to talk about isomorphisms.

The following result holds: $V$ is an operationally closed set, with $W \subset V$, where $V$ is a normal extension of $W$ iff there exists a base of $V / W, a_{\lambda}$, and an irreducible operationally definable relation $\rho$ in terms 
of $W$ such that $\rho\left(a_{\lambda}\right)$, and any solution of $\rho$ is composed by elements of $V$.

Various results of abstract Galois theory, as developed in da Costa, Rodrigues (2007), remain valid in the present situation, with convenient adaptations. This is the case, for instance, of the following three propositions, whose proofs are similar to the proofs of the analogous theorems in da Costa, Rodrigues (2007):

Theorem 3.1 Let $e=\left\langle D, o_{\iota}\right\rangle$ be the structure considered above; $W$ and $V$ are two sets such that $W \subset V \subset D$, and $V$ is operationally closed; let $a_{\lambda}$ be a base of $V / W$ and $\rho$ an operationally irreducible relation operationally definable in terms of $W$, verified by $a_{\lambda}$. Under these hypotheses, any isomorphism $\theta$ of $V$ on the set $V^{\prime} \subset D$ that leaves the elements of $W$ invariant is a bijection of $V$ on $V^{\prime}$, such that if $v \in V$ and $v=\phi_{v}\left(a_{\lambda}\right)$, then $\theta v=\phi_{v}\left(b_{\lambda}\right)$, where $b_{\lambda}$ is a solution of $\rho$, determined by $\theta$, and conversely.

Theorem 3.2 Under the conditions of the previous theorem, if $V$ is a normal extension of $W$ and the relation

$$
\exists y_{\lambda}\left(x_{\lambda}=x \phi_{x_{\lambda}}\left(x, y_{\lambda}\right) \wedge \rho\left(y_{\lambda}\right)\right)
$$

is operationally definable in terms of $W$, then the isomorphisms with domain $V$, that leave the elements of $W$ invariant, transform $V$ on $V$ and are automorphisms of $V$ leaving the members of $W$ invariant.

The relation (1) above appears in the proof of Theorem 6.3 of da Costa, Rodrigues (2007).

Theorem 3.3 In the structure $e=\left\langle D, o_{\iota}\right\rangle$, let $V$ and $d$ be, respectively, an operationally closed subset of $D$ and an element of $D$ that remains invariant under the group of all automorphisms of e that leave the elements of $V$ invariant. Under these conditions, if a certain relation of the same kind as (1) is operationally definable, then $d \in V$. 
When the theory just outlined is restricted to finite separable extensions of fields, the theorem of the primitive element shows that the bases can be constituted by one element, and Tschirnhaus transformations are at our disposal. Relations like (1) are operationally definable, and we are able to prove propositions such as the theorems 8.4 and 8.6 of da Costa, Rodrigues (2007). In this way, by employing properties of fields and their separable extensions, we arrive at classical Galois theory, but in a way very different from that followed by Krasner (outlined in the previous section). (For further details on these issues, see Sebastião e Silva (1985, 1945 and 1985).)

\section{UNIVERSAL THEORY OF STRUCTURES}

Abstract or generalized Galois theory, which can be viewed as expanding universal algebra, is also part of a universal theory of structures that extends further universal algebra to the class of all structures. Bourbaki was the first author to develop, at least syntactically, such a theory (see Bourbaki (1968), chapter IV). We close this paper by making some remarks on the universal theory of structures. To begin with, we note that the definitions of da Costa, Rodrigues (2007) and of the present paper can be simplified if the description symbol $\iota$ is added to our languages as a primitive symbol (with appropriate postulates) or as a defined symbol. For example, it is clear that a relation $r$ is definable in the wide sense in the structures $e$, interpreted in the language $\mathcal{L}$, iff there exists a closed term $\tau$ of $\mathcal{L}$ such that $r$ is denoted by $\tau$.

The closed term $\tau$ of $\mathcal{L}$ is intrinsic (in $e$ ) iff it is invariant under the Galois group of e, i.e., its denotation remains the same under the action of the Galois group. Therefore, a relation $r$ of the scale of $e$ is definable in the wide sense in $e$ iff there exists an intrinsic closed terms $\tau$ such that $\tau$ denotes $r$. 
The structures of Bourbaki (see Bourbaki (1968)) and those of extant mathematics have in general various basic sets; for instance, the field of scalars and the set of vectors in vector spaces. However, it is always possible to reduce this kind of structures to structures with only one basic set, provided some extra predicates (unary relations) are introduced, one for each basic set, with new axioms governing these predicates. The same device can also be applied to species of structures, that is, to axiomatic theories or systems.

Usually, there are two sorts of basic sets: the auxiliary and the principal. When automorphisms of structures are considered, it is supposed that the elements of the auxiliary basic sets remain fixed. This is the case of vector spaces, in which the scalars are invariant under the Galois group of these structures, and the case of real Lie groups whose elements of the Galois group keep the real numbers fixed. In this situation, the Galois group of the structure is restricted by certain conditions, but the central results of the theory of structures remain valid.

The construction of structures and of species of structures employs several procedures, such as: axiomatization, deduction, combination, derivation and the use of universal mappings (see Bourbaki (1968), chapter 4). Axiomatization constitutes the general method to introduce new species of structures. The set-theoretic framework of the structures included in the new species is formulated, and the specific axioms are listed. The axioms, expressed in the language of set theory, must be transportable: they have to be invariant by isomorphism. Sometimes, by extension, there are no specific axioms, and the species has only a set-theoretic counterpart.

The standard concepts of Bourbaki's theory of structures (Bourbaki (1968), chapter 4) are easily treated in our universal theory of structures. For instance, in a topological group, the group law is deducible 
by means of an intrinsic term, which defines the law. We can also deduce the underlying topology.

We combine two or more species of structures to obtain new ones, such as topological groups, differentiable manifolds and fibre bundles. By means of Cartesian product and passage to the quotient, one obtains the so-called derived structures, for instance, initial and final structures. Projective and inductive limits are other means to build structures (see Bourbaki (1968)). As noted in the introduction above, we use an extended set theory (ZFC) with universes. Thus, homological-algebraic, $\mathrm{K}$-theoretic and $\mathrm{C}^{*}$-algebraic constructs can be formulated as well.

Furthermore, the usual model theory is part of the universal theory of structures. Both from the historical and the technical points of view, the works of logicians such as Tarski, Robinson, Gödel, Skolem, and Fräissé, among others, are of fundamental relevance.

All of the developments just described and the literature on Klein's Erlangen Program, including its extensions via action of groups and Cartan's ideas for the systematization of Riemannian geometry, show how relevant the universal structure theory is.

In fact, one of the motivations for the development of Klein's Erlangen Program emerged from the need for systematizing the multiplicity of geometries that, by the end of the 19th century, had been developed. Klein realized that the relations examined by distinct geometries were the invariants of different transformation groups. On his view, there is a general format for the study of problems in geometry, which is connected with the identification of the properties that remain invariant under various transformation groups. He notes:

Let there be given a manifold and a group of transformations in it. [Klein's goal is] to investigate the configurations belonging to the manifold with respect to such properties as remain invariant under the transformations of the group. (Klein (1983), p. 67) 
As Torretti (1999, p. 155, footnote 10) points out, by 'manifold' Klein probably means something somewhat wider than, but still akin to, the sense of 'manifold' formulated via sets (or $n$-tuples of sets) of real, or complex, numbers with the standard topologies inherited from the real, or complex, number fields. According to Klein, the study of the properties that remain invariant under various group transformations

is the universal problem which spans not only ordinary geometry but also, in particular, the new geometric methods to be mentioned later and the different treatments of manifolds with arbitrarily many dimensions. (Klein (1983), p. $67)$

This "universal problem" becomes particularly pressing given the development of different geometries, such as parabolic, hyperbolic and elliptic geometries. The plurality of geometrical systems immediately raises the issue of which of these systems is the "true geometry". Klein's strategy was to develop a mathematical framework in terms of which this issue could be ultimately dissolved. On his view, each geometry has equal right or equal justification (Klein (1983), p. 67). After all, by studying the properties that remain invariant under different group transformations, it is possible to specify systematically the various geometries. As a pure system, each geometry is perfectly acceptable. The issue of its truth does not emerge in this context. (For further discussion of Klein's Erlangen Program and its philosophical significance, see Torretti (1984 and 1999).)

We can say that the truth of a geometry only surfaces in the context of the application of that geometry to the physical world (see da Costa (1997)). In this context, suitable interpretations of the geometrical terms need to be offered so that the geometrical system can be connected to appropriate aspects of the world. It is only then that the issue of the (empirical) truth of a geometrical system can be formulated. 
It is worth noting that analogous points can be made about the universal theory of structures. Similarly to Klein's program, a universal theory of structures (of which abstract Galois theory is part) emphasizes the significance of identifying invariances of group transformations in the characterization of various kinds of structures. Just as Klein's program, a universal theory of structures also provides an overall framework to study mathematical structures systematically. In a clear sense, when the structures in question are geometrical in nature, Klein's program becomes a particular case of abstract Galois theory. Interestingly, abstract Galois theory itself offers a generalization of classical Galois theory, making room for different formulations of the latter. The move toward more abstract formulations, whether of geometrical systems or of mathematical structures, is a significant feature of mathematics from the late 19th century on. The elegance and insight of the resulting approaches to structures is quite telling.

Furthermore, similarly to what happens in geometry, we also have in the case of a universal theory of structures a clear form of pluralism: a pluralism about mathematical structures. As noted above, there is a plurality of structures throughout mathematics, and a universal theory of structure provides a framework to study such structures systematically. And similarly to what happens with Klein's program, the issue of which of these mathematical structures are the "true ones" does not emerge. From the point of view of pure mathematics, every mathematical structure is perfectly acceptable. This does not mean, of course, that each such structure is equally significant or mathematically relevant. Some structures, such as, e.g., group structures, due to their impressive malleability and applicability, have a relevance that transcends the immediate context in which they were originally formulated. And a universal theory of structures provides a conceptual setting to study such structures systematically. 
In our view, all of current classical mathematics can be developed within a universal theory of structures. This is, of course, an old claim, according to which all of classical mathematics is reducible to set theory; a claim that was precisely formulated by Bourbaki. This reduction does not mean that every future result of classical mathematics can be derived from a set theory such as ZFC, but only that the intuitive structures that classical mathematicians currently use are reducible to a universal theory of structures. In other words, current classical mathematics can be unified and systematized by such a universal theory. Note, however, that this does not mean that we have adopted a platonist ontology, given that it is possible to interpret ZFC in a purely symbolic and formal way (see da Costa et al. (2005)). Nor does it mean that classical mathematics can be entirely derived from ZFC, since this would violate Gödel's theorem. We are dealing with a conceptual unification and systematization here.

Another significant aspect of a universal theory of structures is the emphasis it gives to the concept of definability as a central component in the study of mathematical structures. In philosophical discussions about indiscernibility and individuality of quantum particles, for example, in the context of the foundations of physics, it is sometimes assumed that such concepts stand for metaphysical features of the objects under consideration. On this metaphysical conception, the indiscernibility and individuality of a particle is thought of as an objective, language-independent property of that object. As a result, whether a particle is discernible, whether it is an individual or not, does not depend on the language or the logic that is used to describe the particle.

However, it is unclear that the metaphysical conception of indiscernibility and individuality is stable. After all, in order to express the view, in order to formulate it linguistically, we clearly need to use a language and a logic. And as it becomes clear with a universal theory of structures (of which, as we noted, abstract Galois theory is part), 
depending on the resources of the language and the logic we use, the properties of the concepts that can be defined, including those of indiscernibility and individuality, will change. It is then unclear whether it is possible to formulate explicitly the metaphysical conception of an individual. According to a universal theory of structures, since definability is a property that depends on the language and the logic we use, the definability of concepts such as indiscernibility and individuality will also be language- and logic-dependent. Rather than absolute concepts (as the metaphysical conception would have it), indiscernibility and individuality are then relative to the logic and language in question. A significant philosophical re-conceptualization of the issues emerges.

A universal theory of structures thus has applications not only in physics (and in science, more generally), but in particular in the foundations of quantum mechanics (for a discussion of indiscernibility of quantum particles, see Caulton, Butterfield (2008)). (In future papers, we intend to study the concept of structure from the categorial point of view, to develop the universal theory of structures and to present some of its applications to physics.)

\section{REFERENCES}

BOURBAKI, N. Theory of Sets. London-Ontario: Hermann, Addison Werley, 1968.

BRIGNOLE, D., DA COSTA, N. C. A. "On supernormal Ehresmann-Dedecker universes", Math. Z., 122, p. 342-350, 1971.

BUNINA, E. I., ZAKHAROV, V. K. "Characterization of model Mirimanov-von Neumann cumulative sets". J. Math. Sci., 138, p. 5830-5891, 2006.

CAULTON, A., BUTTERFIELD, J. "On kinds of indiscernibil- 
ity in logic and metaphysics" preprint, Cambridge University, 2008.

DA COSTA, N. C. A. "Modéles et univers de Dedecker", $C . R$. Acad. Sc. Paris, 275, p. 483-486, 1972.

- Logiques classiques et non classiques. Paris: Masson, 1997.

DA COSTA, N. C. A.; BUENO, O., FRENCH, S. "A coherence theory of truth", Manuscrito, 29, p. 263-290, 2005.

DA COSTA, N. C. A., RODRIGUES, A. A. M. "Definability and invariance, Studia Logica, 86, p. 1-30, 2007.

DAVEY, B. A., PRIESTLEY, H. A. Introduction to Lattices and Order. Cambridge: Cambridge University Press, 2002.

DENEKE, K., ERNÉ, M., WISMATH, S. L. (eds.) Galois Connections and Applications. Dordrecht: Kluwer Academic Publishers, 2004.

ERNÉ, M., KOSLOWSKI, J., MELTON, A., STRECKER, G. E. "A primer on Galois connections", Annals of the New York Academy of Sciences, 704, p. 103-125, 1993.

GALATOS, N., JIPSEN, P., KOWALSKI, T., ONO, H. Residuated Lattices: An Algebraic Glimpse at Substructural Logics. Amsterdam: Elsevier, 2007.

GIERZ, G., HOFMANN, K., KEIMEL, K., LAWSON , J., MISLOVE, M., SCOTT, D. Continuous Lattices and Domains. Cambridge: Cambridge University Press, 2002.

KLEIN, F. "Vergleichende Betrachtungen uber neuere geometrische Forschungen", Mathematische Annalen, 43, p. 63-100, 1893. 
KRASNER, M. "Une généralisation de la notion de corps", Journal de Math. Pures et Appl., 17, p. 367-385, 1938.

—_ "Généralisation abstraite de la théorie de Galois", Algébre et Théorie des Nombres, 24, p. 163-168, 1950.

- "Endothéorie de Galois abstraite", Séminaire DubreilPisot, Alg. et Théorie des Nombres, 22, 6, 19pp, 1968-69.

—. "Abstract Galois theory", preprint, University of Paris VI, Paris, 34pp, 1973.

—. "Polythéorie de Galois abstraite dans le cas infini general", Ann. Sc. Univ. Clermont, Sér. Math., 13, p. 87-91, 1976 .

MAC LANE, S. Categories for the Working Mathematician. (Second edition.) New York: Springer, 1998.

MARSHALL, M. V., CHUAQUI, R. "Sentences of type theory: the only sentences preserved under isomorphisms", Journal of Symbolic Logic, 56, p. 932-948, 1991.

PICADO, J. "The quantale of Galois connections", Algebra Universalis, 56, p. 527-540, 2005.

ROGERS Jr., H. "Some problems of definability in recursive function theory", in J. N. Crossley (ed.), Sets, Models and Recursion. Amsterdam: North-Holland, p. 183-201, 1996.

SHOENFIELD, J. R. Mathematical Logic. Reading-London: Addison-Wesley, 1967.

SEBASTIÃO E SILVA, J. "Para Uma Teoria Geral Dos Homomorfismos (Thesis)", in J. C. Ferreira, J. S. Guerreiro J. S. Oliveira, Obras de José Sebastião e Silva, Volume 1. Lisbon: Sociedade Portuguesa de Matemática, 1985. 
- "Sugli automorfismi di un sistema mathematico qualunque", Comm. Pontif. Acad. Sci., 9, p. 327-357, 1945.

—. "On automorphisms of arbitrary mathematical systems", History and Philosophy of Logic, 6, p. 91-116, 1985.

TARSKI, A. Logic, Semantics, Metamathematics. (Edited by John Corcoran.) Indianapolis: Hackett, 1983.

TORRETTI, R. Philosophy of Geometry From Riemann to Poincaré. Dordrecht: Reidel, 1984.

- Philosophy of Physics. Cambridge: Cambridge University Press, 1999.

ZAKHAROV, V., BUNINA, E., MIKHALEV, A., ANDREEV, P. "Local theory of sets as a foundation for category theory and its connection with Zermelo-Fraenkel set theory", J. Math. Sci., 138, p. 5763-5829, 2006. 